\title{
Electromagnetic energy transport along arrays of closely spaced metal rods as an analogue to plasmonic devices
}

\author{
Stefan A. Maier, Mark L. Brongersma, and Harry A. Atwater ${ }^{a}$ \\ Thomas J. Watson Laboratory of Applied Physics, California Institute of Technology, \\ Pasadena, California 91125
}

(Received 21 July 2000; accepted for publication 9 November 2000)

\begin{abstract}
The transport of electromagnetic energy along structures consisting of arrays of closely spaced metal rods ( spacing $=0.2 \mathrm{~cm}$ ) was investigated in the microwave regime at $8.0 \mathrm{GHz}(\lambda=3.7 \mathrm{~cm})$. The dispersion relation shows that information transport occurs at a group velocity of $0.6 \mathrm{c}$. The electromagnetic energy is highly confined to the arrays $(90 \%$ within a distance of $0.05 \lambda$ from the array). The propagation loss in a straight array is $3 \mathrm{~dB} / 8 \mathrm{~cm}$. Routing of energy around $90^{\circ}$ corners is possible with a power loss of $3-4 \mathrm{~dB}$. Analogies to plasmon wires consisting of arrays of nm-size metal clusters are discussed. (C) 2001 American Institute of Physics. [DOI: 10.1063/1.1337637]
\end{abstract}

In a recent paper, a method was proposed for the guiding and switching of visible light using plasmon wires, which consist of arrays of closely spaced metal nanoparticles. ${ }^{1,2}$ Whereas an optical mode in conventional optical waveguides has a minimum size of $\lambda / 2 n$ (above the diffraction limit of light), plasmon wires do not suffer from this limitation. Furthermore, it was shown that light can be guided efficiently around sharp corners (bending radius $\ll \lambda$ ) akin to photonic crystal structures, ${ }^{3}$ in contrast to conventional waveguide technology.

As a large-scale analogue to nanoscale plasmonic devices that operate in the visible part of the electromagnetic (EM) spectrum, in this letter we discuss the propagation of EM energy along $\mathrm{cm}$-scale periodic structures in the radiofrequency (rf) regime, similar to linear Yagi antenna arrays. ${ }^{4}$ The geometry of such an array is pictured in the inset of Fig. 1. It consists of a dipole source and a series of equally spaced metal rods with a height $h$ and an inter-rod spacing $s$. It is well known experimentally ${ }^{5}$ and theoretically ${ }^{6,7}$ that such arrays show guiding properties as long as $h / \lambda_{F}<0.5$, where $\lambda_{F}$ is the free-space wavelength of the EM wave emitted by the source. $^{4-7}$ Under this condition, the phase velocity of the guided wave is less than the free-space velocity $c$. Conventional Yagi antenna arrays are aimed at radiating out EM energy into the far field and have inter-rod spacings on the order of $\lambda_{F} / 4$. In this letter, we will describe structures with a significantly smaller spacing of $\lambda_{F} / 20$, which allow for a more efficient guiding of energy around sharp corners. A smaller spacing between adjacent rods results in a slower phase velocity ${ }^{6,7}$ and a stronger confinement of the EM waves to the structure due to an increased near-field coupling. We describe EM simulations and experiments that examine the propagation of energy in straight arrays, sharp corners, and tee structures. We will conclude by discussing the link to nm-sized plasmon wire structures.

Three structures consisting of arrays of $\mathrm{Cu}$ rods were investigated, namely, a straight line, a corner, and a tee structure. The rods have a diameter of $1 \mathrm{~mm}\left(d=0.002 \lambda_{F}\right)$, a height of $1.4 \mathrm{~cm}\left(h=0.37 \lambda_{F}\right)$, and were spaced $2 \mathrm{~mm}$ apart

${ }^{a)}$ Electronic mail: haa@daedalus.caltech.edu $\left(s=0.05 \lambda_{F}\right)$. They were fixed in position using a Styrofoam construction that showed negligible guiding properties. The first rod was a center-fed dipole driven at $8.0 \mathrm{GHz}$ using a HP $8620 \mathrm{C}$ sweep oscillator. The distribution of the EM field along the array was studied by local power measurements using a 1-cm-long probe dipole and a HP 8472 crystal detector connected to a HP $415 \mathrm{E}$ power meter. These measurements were performed at a distance of $0.15 \lambda_{F}$ from the array to avoid significant interference with the propagating EM wave. None of the structures were terminated with impedance matching loads to obtain standing-wave patterns for physical analysis. Full-field EM simulations were performed on these structures using antenna simulation software. ${ }^{8}$ In the simulation, the axis of the rods were chosen to be in the $z$ direction and the center of the first rod (source) is located at $(x, y, z)=(0,0,0)$.

Figure 1 shows the dispersion relation for EM waves propagating on a linear array of 101 copper rods determined by EM simulations at various frequencies from 6.0 to 9.5 $\mathrm{GHz}$. The decrease in slope with increasing wave vector $k$

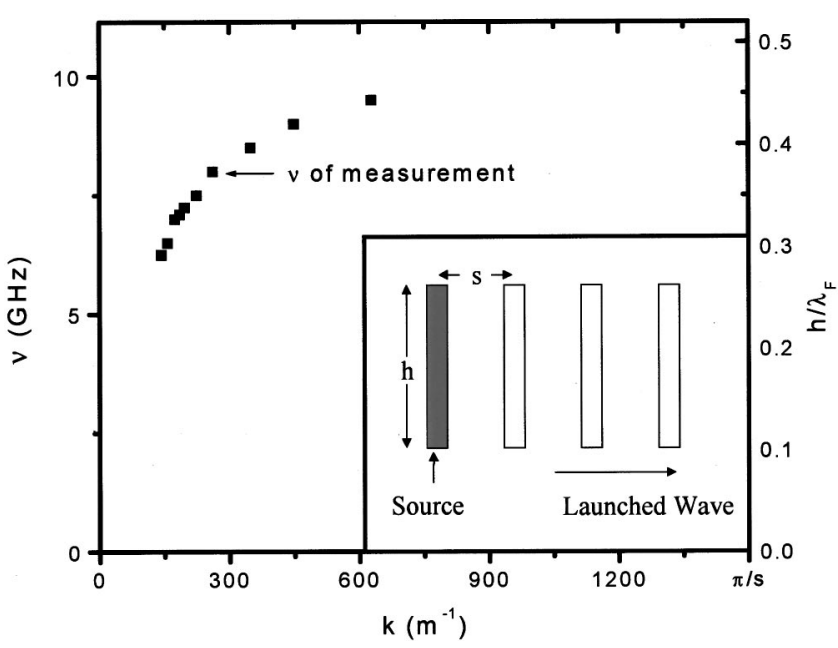

FIG. 1. Dispersion relation for EM waves propagating on a linear Yagi array consisting of $101 \mathrm{Cu}$ rods obtained by EM simulations. The rods have a diameter $d$ of $1 \mathrm{~mm}$, a height $h$ of $1.4 \mathrm{~cm}$, and were spaced by $s$ $=2 \mathrm{~mm}$ apart. The inset shows a schematic of such a Yagi array including a source element. 


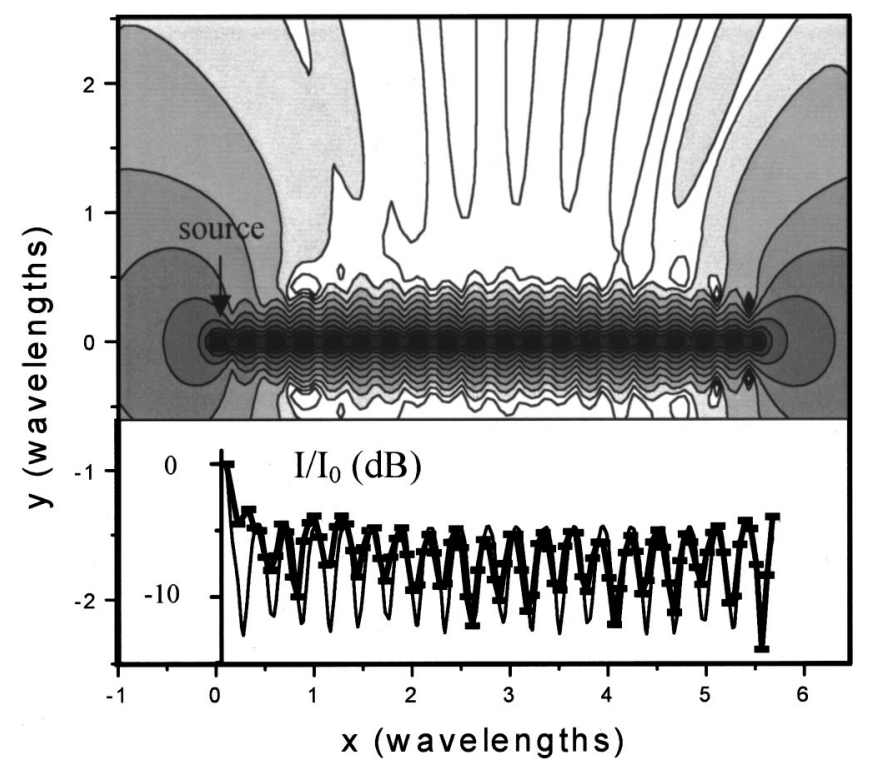

FIG. 2. Distribution of the absolute value of the $z$ component of the electricfield vector $\left|E_{z}\right|$ in the $x-y$ plane for a linear array of 101 rods. The rods are $1 \mathrm{~mm}$ in diameter, $1.4 \mathrm{~cm}$ long, and spaced $2 \mathrm{~mm}$ apart. The gray scale is exponential in the magnitude of $\left|E_{z}\right|$ and spans about $10^{4}$ orders of magnitude. The inset shows both the measured (squares) and simulated (thin solid line) power at a distance of $0.15 \lambda$ from the array.

indicates a decrease of the group velocity of the EM wave. Near the zone boundary at $\pi / s$ the ratio $h / \lambda_{F}$ approaches 0.5 , as predicted by linear antenna theory. ${ }^{6,7}$ At the excitation frequency of $8.0 \mathrm{GHz}$ the phase velocity is $0.65 c$.

Figure 2 shows the distribution of the absolute value of the $z$ component of the electric-field vector $\left|E_{z}\right|$ in the $x-y$ plane for a linear array of 101 rods. The gray scale in this field plot is exponential in the magnitude of $\left|E_{z}\right|$ and spans four orders of magnitude. The inset shows both the measured (squares) and simulated (thin solid line) power at a distance of $0.15 \lambda_{F}$ from the array, normalized to the power obtained at the position of the source. Most of the energy emitted by the source is lost due to radiation into the far field, as seen in the field plot and in the inset as an initial drop in the measured power after rod number 1. A standing-wave pattern is recognized, indicating weak attenuation along the array and strong reflection at the end points, as will be quantified. The period of the standing wave obtained by simulation and experiment are in excellent agreement. The discrepancies in the measured and simulated power can, in part, be attributed to the finite diameter of the probe dipole, which causes an averaging out of the sharp features seen in the simulation. About $90 \%$ of the energy is confined within a distance of $0.05 \lambda_{F}$ from the array, demonstrating strong guiding of the EM wave by the array. The propagation loss $\alpha$ of the EM wave and its reflection coefficient $R$ can be estimated by assuming that the standing-wave pattern results from EM waves that travel back and forth along the array. From such an analysis we determined that $\alpha \approx 3 \mathrm{~dB} / 8 \mathrm{~cm}$ and $R$ $\approx 75 \%$. The remaining $25 \%$ of the power is radiated into the far field at the end of the array, as can be seen in the field plot. It is important to note that radiation loss only occurs at the two ends of the linear array. More generally, radiation loss occurs at each discontinuity in the structure where the EM wave changes direction. For the design of more complex Downloaded 03 Apr 2006 to 131.215.225.171. Redistribution subje

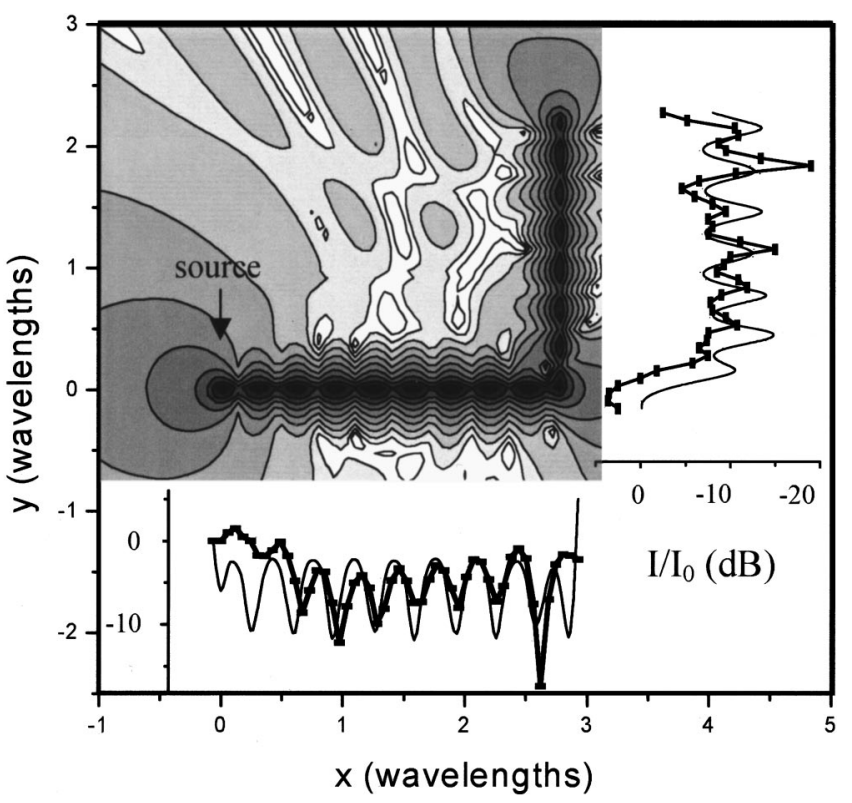

FIG. 3. Distribution of $\left|E_{z}\right|$ in the $x-y$ plane for a corner structure consisting of two linear arrays of rods that make a $90^{\circ}$ angle. The rods are $1 \mathrm{~mm}$ in diameter, $1.4 \mathrm{~cm}$ long, and spaced $2 \mathrm{~mm}$ apart. The gray scale is exponential in the magnitude of $\left|E_{z}\right|$ and spans about $10^{4}$ orders of magnitude. The inset shows both the measured (squares) and simulated (thin solid line) power at a distance of $0.15 \lambda$ from the array on the outside of the corner.

array structures it is important to quantify the losses at such discontinuities. For this purpose, we have investigated the transport around $90^{\circ}$ corners.

Figure 3 shows the distribution of $\left|E_{z}\right|$ in the $x-y$ plane for a corner structure consisting of two linear arrays of rods that make a $90^{\circ}$ angle. The gray scale is exponential in the magnitude of $\left|E_{z}\right|$ and spans four orders of magnitude. The inset shows both the measured and simulated power at a distance of $0.15 \lambda_{F}$ on the outside of the corner, normalized to the power obtained at the position of the source. As for the straight line, simulation and experiment are in reasonable agreement. As expected, power is lost in turning the corner due to radiation into the far field. The ratio of the power in the sidearm to the power in the main arm is $3-4 \mathrm{~dB}$. The decrease in power is due to radiation and back reflection at the corner. We also examined a tee structure in which an EM wave was injected into the stem and splitted into the two side arms. The ratio of the power in one side arm to the power in the stem was determined to be of about $8 \mathrm{~dB}$. This is roughly twice the power lost in the corner structure. At present, it is investigated whether transmission around corners can be optimized by placement of additional rods that frustrate radiation into the far field and reduce back reflection.

Another discontinuity that is worth mentioning is the breaking of the perfect periodicity in the array. It was found that the guiding properties of these arrays are not significantly altered when rods are displaced by as much as $50 \%$ of the inter-rod spacing from their original positions. Simulations show that straight arrays with random displacements of a subset of the rods still demonstrate strong guiding and comparable attenuation. If near-field coupling would be dominant, the transport would be extremely sensitive to a breaking of the perfect translation symmetry, because the strength of near-field coupling is strongly dependent on the spacing $\left(\propto 1 / s^{3}\right)$. This strong distance dependence is well to AIP license or copyright, see http://apl.aip.org/apl/copyright.jsp 
known from Förster resonance energy transfer. ${ }^{9}$ It is thus concluded that the intermediate-field and far-field terms play an important role in the transport. For this reason, fabrication-induced deviations from perfect translation symmetry in array structures can be tolerated.

At this point, it is worthwhile to note a few analogies and differences between cm-scale metal-rod arrays and plasmon wires, consisting of nm-size noble metal clusters. ${ }^{1}$ For the excitation of EM waves in metal-rod arrays, we used a rf dipole antenna. Arrays of metal clusters can efficiently be excited near the surface plasmon resonance frequency. ${ }^{10}$ For $\mathrm{Au}, \mathrm{Ag}$, and $\mathrm{Cu}$ clusters, this resonance occurs at optical frequencies and is associated with a resonantly enhanced absorption cross section. Due to the enhanced absorption cross section, a strong coupling between the metal particles can be obtained even for cluster sizes that are small compared to the interparticle spacing. If $\mathrm{nm}$-size spherical clusters with a diameter $d$ are used, a strongly guiding array can be realized for which $d<s \ll \lambda_{F}$. This is a necessary requirement for near-field coupling to be the only contributor to the transport. Since this condition is met in plasmon wires, far-field radiation at discontinuities is negligible. ${ }^{1}$ This situation cannot be obtained in the rf regime where the absorption cross section of the rods is not resonantly enhanced. In the rf strong guiding can only be obtained if the height of the rods are a substantial fraction of $\lambda_{F} / 2,{ }^{4}$ and thus $h<s \ll \lambda_{F}$ cannot hold. The positive effect of an increase in the absorption cross section near the plasmon resonance frequency is counteracted by a concomitant increase in the attenuation due to resistive heating to about $3 \mathrm{~dB} / \lambda_{F}{ }^{1}$ This limits the size of functional plasmonic devices to a few $\lambda_{F}$. It has been dem- onstrated theoretically that functionality can indeed be obtained at this length scale. ${ }^{1}$

In conclusion, we have shown by experiment and simulation that in the rf regime EM waves can be guided along three-dimensional structures consisting of closely spaced metal rods. Significant losses mainly occur when the wave changes direction due to radiation into the far field and reflections. It is anticipated that these radiation losses will be negligible for plasmon wire structures operating in the optical regime and transporting EM energy by near-field coupling. Such structures could potentially be among the smallest structures with optical functionality.

The authors would like to thank Bill Bridges for useful discussions and for letting them use his microwave laboratory at Caltech. This work was sponsored by the National Science Foundation.

\footnotetext{
${ }^{1}$ M. L. Brongersma, J. W. Hartman, and H. A. Atwater, Phys. Rev. B (to be published).

${ }^{2}$ M. Quinten, A. Leitner, J. R. Krenn, and F. R. Aussenegg, Opt. Lett. 23, 1331 (1998).

${ }^{3}$ A. Mekis, J. C. Chen, I. Kurland, S. Fan, P. R. Villeneuve, and J. D. Joannopoulos, Phys. Rev. Lett. 77, 3787 (1996).

${ }^{4}$ J. O. Spector, J. IEE 105, 38 (1958).

${ }^{5}$ J. Brown and J. O. Spector, Proc. IEE 104, 27 (1957).

${ }^{6}$ D. L. Sengupta, IRE Trans. Antennas Propag. AP-7, 234 (1959).

${ }^{7}$ F. Serracchioli and C. A. Levis, IRE Trans. Antennas Propag. AP-7, 424 (1959).

${ }^{8}$ EZNEC v. 2.0, R. W. Lewallen (1997-1999).

${ }^{9}$ Th. Förster, Ann. Phys. 2, 55 (1948).

${ }^{10} \mathrm{U}$. Kreibig and M. Vollmer, Optical Properties of Metal Clusters (Springer, Berlin, 1994).
} 